Methods We evaluated in vitro STA551's effect on IFN- $\gamma$ production from human CD8 $+\mathrm{T}$ cells. We also evaluated in vivo STA551's effect on tumor growth, RNAseq-based immunerelated gene expression, immunohistochemistry, and $\mathrm{T}$ cell activation in tumor and non-tumor tissues in human CD137 knock-in mice treated with mouse surrogate STA551 (Sta-MB) or urelumab (Ure-MB) in combination with anti-PD-L1 antibody.

Results In a human T cell assay, STA551 induced IFN- $\gamma$ only in the presence of ATP. In contrast, urelumab induced IFN- $\gamma$ regardless of ATP concentration. In mice with Colon 38 tumors, Sta-MB inhibited tumor growth at least as strongly as Ure-MB, but whereas Ure-MB elicited systemic immune responses in draining lymph node, spleen, and liver, Sta-MB appeared to evade such responses. To confirm immune responses in tumors, we evaluated immune-related gene expression and found changes after treatment with Sta-MB or Ure-MB. These results suggest that STA551 works only in tumor tissue. Furthermore, Sta-MB with anti-PD-L1 antibody synergistically inhibited tumor growth and dramatically changed immune-related gene expression, CD8 $+\mathrm{T}$ cell infiltration, and PD-L1 expression without systemic immune responses. Also, it was well-tolerated in cynomolgus monkey in a repeated-dose toxicity study*.

Conclusions STA551 is a novel anti-CD137 agonist antibody that exerts agonistic activity selectively in tumors without ontarget toxicity in non-tumor tissues, regardless of tumor-associated antigen expression. These results strongly support the clinical testing of STA551 for the treatment of solid tumors. STA551 is currently being tested in a phase 1 clinical study. Ethics Approval All animal studies were reviewed and approved by the Institutional Animal Care and Use Committee (IACUC).

http://dx.doi.org/10.1136/jitc-2020-SITC2020.0578

\section{LACTATE DEHYDROGENASE C-ASSOCIATED MOLECULAR NETWORKS PREDICT ENHANCED TUMOR GROWTH AND IMPAIRED IMMUNE RESPONSE IN BREAST CANCER}

Julie Decock* , Adviti Jana. Qatar Biomedical Research Institute (QBRI), Doha, Qatar

Background Cancer testis antigens (CTAs) have gained interest in the field of anti-cancer therapy as they offer the opportunity to target tumor cells with little off/on-target side effects given their restricted expression patterns. Several CTAs have been implicated as mediators of cancer hallmarks including cancer metabolism, proliferation, survival, and cell motility. Lactate dehydrogenase C (LDHC) expression has been observed in various cancer types and likely confers a survival advantage to tumor cells through metabolic reprograming. Thus, targeting LDHC has the potential to inhibit tumor growth and release the anti-tumor immune response from the acidic immunosuppressive microenvironment. This study aimed to explore the changes in the transcriptome of breast cancer cells upon in vitro LDHC targeting.

Methods We silenced LDHC expression in two breast cancer cell lines (BT549, HCC1954) and investigated the downstream effects on the tumor cell transcriptome. In addition, differentially expressed genes were subjected to regulatory network analyses and expression of key regulators was interrogated in the TCGA breast cancer dataset.

Results We identified 47 up- and 55 down-regulated transcripts after LDHC silencing $(2.0$-fold change, adj $\mathrm{p}<0.05)$.
Specifically, we found that LDHC expressing breast cancer cells display an enrichment of genes involved in canonical pathways regulating cell cycle checkpoint control, BRCA1mediated DNA damage response and NF-kb signaling in response to infection, which is in line with some of our unpublished work. In support, downstream effector analysis demonstrate that LDHC silencing negatively affects biological functions such as cellular development, cellular growth and proliferation, cell migration and cell infiltration. Upstream regulator analyses revealed that the observed changes in gene expression are associated with $\operatorname{mTOR}(p=1.27 \mathrm{e}-5, \mathrm{z}=2.242)$ and CASP3 $(p=3.2 \mathrm{e}-4, \mathrm{z}=2.250)$ mechanistic networks, which in the presence of LDHC are predicted to activate TP53, Myc, NF-KB complex, STAT1/3, PRKC, CDK2, FOXO3 and HIF-1a while inhibiting SMAD3, PTEN, ATM, IL18 and BCL2. Furthermore, causal network analysis revealed a higherlevel regulation by miR378a-3p $(\mathrm{p}=1.4 \mathrm{e}-7, \mathrm{z}=-3.117)$, affecting the mechanistic networks and ultimately promoting tumor cell viability and proliferation, tumor cell movement and cell cycle progression in LDHC expressing cells. Interestingly, the miR378a causal network also indicated inhibition of the immune response in LDHC positive cells. Correlation analysis using the TCGA breast cancer dataset indicated a weak correlation between LDHC expression and the mechanistic regulator mTOR $(\mathrm{R}=0.26, \mathrm{p}=1.82 \mathrm{e}-18)$.

Conclusions Our findings demonstrate that therapeutic targeting of LDHC may inhibit tumor growth while releasing the anti-tumor immune response in breast cancer, and warrant further in-depth investigation.

Acknowledgements This work was supported by a grant from the Qatar Biomedical Research Institute (grant number VR94), awarded to Dr Julie Decock.

http://dx.doi.org/10.1136/jitc-2020-SITC2020.0579

\section{HIGH DOSE-RATE BRACHYTHERAPY OF LOCALIZED PROSTATE CANCER CONVERTS TUMORS FROM COLD TO HOT}

Simon Keam*, Heloise Halse, ThuNgoc Nguyen, Minyu Wang, Nicolas Van Kooten Losio, Catherine Mitchell, Franco Caramia, David Byrne, Sue Haupt, Georgina Ryland, Phillip Darcy, Shahneen Sandhu, Piers Blombery, Ygal Haupt, Scott Williams, Paul Neeson. Peter MaCallum Cancer Center, Melbourne, Australia

Background Prostate cancer is frequently cured with high dose-rate brachytherapy (HDRBT) radiation as a front-line treatment. Although considered to be an immune-excluded tissue, immune responses to radiation are implicated in driving tumour-eradication in prostate cancer. ${ }^{1}$ This has not been proven, and yet is used as the rationale for clinical trials combining radiation and immunotherapies. ${ }^{2}$ We hypothesise that there is a predictable relationship between radiation and the immune responses in prostate cancer that could be used to provide sound rationale for specific immune interventions in solid tumours that are made possible by radiation therapy.

Methods We present here new results stemming from our recently published immunoprofiling study of world-unique preand post-radiation tissues from 24 prostate cancer patients (figure 1A), RadBank cohort). ${ }^{3}$ These samples were assessed using immune cell multiplex IHC, gene expression profiling, digital spatial profiling (DSP) and computational analysis of cell distribution.

Results This study unequivocally revealed that high dose-rate radiation converts predominately 'cold' prostate tumour tissue 